\title{
Effective conductivities of heterogeneous media containing multiple inclusions with various spatial distributions
}

\author{
H. L. Duan, ${ }^{1,2}$ B. L. Karihaloo, ${ }^{2, *}$ J. Wang, ${ }^{1}$ and X. $\mathrm{Yi}^{1}$ \\ ${ }^{1}$ LTCS and Department of Mechanics and Engineering Science, Peking University, Beijing 100871, P. R. China \\ ${ }^{2}$ School of Engineering, Cardiff University, Queen's Buildings, The Parade, Cardiff CF24 3AA, United Kingdom \\ (Received 29 November 2005; revised manuscript received 25 January 2006; published 5 May 2006)
}

\begin{abstract}
A scheme is proposed for predicting the effective conductivities of heterogeneous media containing ellipsoidal inclusions of diverse shapes, spatial distributions, and orientations. This scheme yields explicit expressions for the effective conductivity tensor in terms of three microstructural parameters that characterize the shape, distribution, and orientation of the inclusions. By expanding the effective conductivity tensor in terms of the volume fraction of the inclusions, it is found that the effect of the shape of the distribution ellipsoid on the effective conductivity tensor is of a higher order in the volume fraction than the effect of the shape of the inclusions. The scheme proposed here generalizes the Maxwell formula to heterogeneous media containing multiple inclusions while also taking into account the orientation of the inclusions. Thus, the existing formulas in the literature are special cases of the general formulas given by the present scheme. The predicted effective conductivities of heterogeneous media containing aligned ellipsoidal inclusions, randomly oriented ellipsoidal inclusions, spheroidal inclusions with orientational distributions, and mixtures of cavities and cracks are found to agree well with the experimental results and the results of other schemes.
\end{abstract}

DOI: 10.1103/PhysRevB.73.174203

PACS number(s): 44.10.+i, 77.84.Lf, 72.80.Tm, 05.60.Cd

\section{INTRODUCTION}

The prediction of the physical properties (e.g., the dielectric constant, the thermal or electrical conductivity, and the effective elastic constants, etc.) of heterogeneous media has been the subject of scientific and engineering interest spanning more than a century. It has attracted the attention of such luminaries as Maxwell, Rayleigh, and Einstein. ${ }^{1-3}$ Many predictive schemes have been well documented. ${ }^{4-10}$ Among these schemes, the Maxwell formula ${ }^{1}$ for the conductivity of a heterogeneous medium containing a very dilute suspension of spheres is the earliest and most well-known one. Since then, the corresponding formulas for the conductivities of media containing ellipsoids ${ }^{11}$ and parallel circular cylinders ${ }^{12}$ have been developed. In the current literature, the Maxwell formula for spheres and Fricke's expressions for ellipsoids are the results of the so-called Maxwell-Garnett effective medium theory. ${ }^{13}$ These results hold for very low volume fractions of the dispersed constituents. Recently, Giordano $^{14}$ predicted the effective permittivities of twophase heterogeneous media containing aligned or randomly oriented ellipsoidal inclusions by using the Maxwell formula for ellipsoids and Bruggeman's scheme. ${ }^{15}$ However, the results of Giordano ${ }^{14}$ only apply to two-phase heterogeneous media, and the results are implicit. Shafiro and Kachanov ${ }^{16}$ predicted the anisotropic effective conductivity of a medium with nonrandomly oriented ellipsoidal inclusions and analyzed the effect of the orientation of inclusions on the effective conductivity. Their results only apply to very dilute concentrations of inclusions and they did not consider the effect of the distribution of inclusions. Therefore, although Maxwell's theory has been extended to two-phase heterogeneous media with ellipsoidal inclusions, ${ }^{11,14}$ it is not yet available for heterogeneous media containing multiple inclusions, nor has any consideration been given to the orientation of the inclusions, especially at nondilute concentrations.

The conduction properties of heterogeneous media depend strongly on their microstructures. Ideally, a predictive scheme should employ general microstructural information to make accurate property predictions. Hashin and Shtrikman ${ }^{17}$ gave the lower and upper bounds on the effective conductivity of a multiphase heterogeneous medium by introducing a homogeneous comparison material. The Hashin-Shtrikman bounds rely only on the properties and volume fractions of the constituents of a heterogeneous medium. With increased microstructural information, e.g., taking into account the information on the relative inclusion location distribution to include point-to-point correlations of order higher than two, the bounds on the effective conductivity can be narrowed, increasing further the precision of the prediction they provide. ${ }^{8,10,18}$

Generally, the effective properties of a heterogeneous medium are affected by three factors, namely, the locations (spatial distribution), the orientations, and the shapes of inclusions. Some researchers have studied the effect of one or two of these three factors on the effective conductivities. For example, the effect of the shape of the inclusions on the effective dielectric constants and optical properties of heterogeneous media has been studied. ${ }^{19-21}$ Willis ${ }^{22}$ predicted the effective conductivities of heterogeneous media by taking into account inclusion shape and spatial distribution jointly through an integral of two-point correlation functions of the medium, but the two ellipsoids describing the inclusion shape and the spatial distribution have to be identically shaped and aligned. To the authors' knowledge, at present, there is no scheme available to predict the joint effect of the above three factors on the effective conductivities of heterogeneous media.

In this paper, we use a variational principle based on homogeneous comparison materials to propose a scheme for 
predicting the effective conductivities of ergodic (statistically homogeneous media) heterogeneous media containing multiple inclusions. This scheme can predict the effective conductivity of heterogeneous media containing ellipsoidal inclusions of diverse shapes and orientations whose centers are distributed with ellipsoidal symmetry. The asymptotic expansions of the resulting effective conductivity tensor in terms of the volume fraction of the inclusions show that the shape of the inclusions affects the effective conductivity through the first order of the volume fraction, but the shape of the distribution ellipsoid affects the effective conductivity through the second order. If the terms higher than the first order in the volume fraction are neglected, the present scheme reduces to the dilute solution of Shafiro and Kachanov. ${ }^{16}$ The formulas so obtained can be regarded as the generalization of the Maxwell formula to media containing multiple inclusions that take into account the effect of the orientation of the inclusions. The present scheme has two distinguishing features: it takes into account the distribution of the inclusions in the heterogeneous media, and the resulting formulas are completely explicit and easy to use; for two-phase heterogeneous media containing aligned ellipsoidal inclusions or randomly oriented ellipsoidal inclusions, while the numerical predictions of the present scheme are very close to those of Giordano, ${ }^{14}$ the predictive formulas are explicit and concise.

\section{BASIC FORMULATION}

As pointed out by Hashin and Shtrikman, ${ }^{17}$ the problems of the prediction of the effective magnetic permeability, dielectric constant, electrical conductivity, thermal conductivity, and diffusivity of heterogeneous media are mathematically analogous. Thus, we consider an electrical conduction problem of a heterogeneous medium. For a representative volume element (RVE) of an ergodic heterogeneous medium with volume $V$, let $\mathbf{q}(\mathbf{x})$ denote the local flux at position $\mathbf{x}$, $\mathbf{H}(\mathbf{x})$ denote the local intensity field, and $\boldsymbol{\Phi}(\mathbf{x})$ the potential field. The local flux and intensity fields within $V$ can be obtained by solving the so-called local problem described by the following basic equations:

$$
\begin{gathered}
\boldsymbol{\nabla} \cdot \mathbf{q}(\mathbf{x})=0, \quad \boldsymbol{\nabla} \times \mathbf{H}(\mathbf{x})=\mathbf{0}, \quad \mathbf{H}(\mathbf{x})=-\boldsymbol{\nabla} \boldsymbol{\Phi}(\mathbf{x}), \\
\mathbf{q}(\mathbf{x})=\boldsymbol{\mu}(\mathbf{x}) \cdot \mathbf{H}(\mathbf{x}) \quad \text { or } \quad \mathbf{H}(\mathbf{x})=\zeta(\mathbf{x}) \cdot \mathbf{q}(\mathbf{x}),
\end{gathered}
$$

where $\boldsymbol{\mu}(\mathbf{x})$ and $\boldsymbol{\zeta}(\mathbf{x})$ are the second-order conductivity and resistivity tensors, respectively. Generally, two kinds of boundary conditions are considered on the boundary $\partial V$ of the RVE,

$$
\boldsymbol{\Phi}(\mathbf{x})=-\mathbf{H}^{0} \cdot \mathbf{x} \quad \text { or } \quad \mathbf{q}(\mathbf{x}) \cdot \mathbf{M}(\mathbf{x})=\mathbf{q}^{0} \cdot \mathbf{M}(\mathbf{x}),
$$

where $\mathbf{H}^{0}$ and $\mathbf{q}^{0}$ are the constant intensity and flux, respectively, and $\mathbf{M}(\mathbf{x})$ is the unit vector normal to the boundary $\partial V$.

Assume that the heterogeneous medium is made up of $N$ homogeneous phases with volumes $V^{(r)}(r=1, \ldots, N)$, whose distributions are defined by characteristic functions $\chi^{(r)}(\mathbf{x})$ (equal to 1 if $\mathbf{x}$ is in phase $r$ and 0 otherwise). The local conduction properties of the linear heterogeneous medium are described by its conductivity and resistivity tensors:

$$
\boldsymbol{\mu}(\mathbf{x})=\sum_{r=1}^{N} \chi^{(r)}(\mathbf{x}) \boldsymbol{\mu}^{(r)}, \quad \zeta(\mathbf{x})=\sum_{r=1}^{N} \chi^{(r)}(\mathbf{x}) \zeta^{(r)} .
$$

Let $\langle\cdot\rangle^{(r)}$ and $\langle\cdot\rangle$ denote spatial averages over $V^{(r)}$ and $V$, respectively, e.g.,

$$
\begin{aligned}
&\langle\mathbf{H}\rangle^{(r)}= \frac{1}{V^{(r)}} \int_{V^{(r)}} \mathbf{H}(\mathbf{x}) d \mathbf{x}, \\
&\langle\mathbf{H}\rangle=\frac{1}{V} \int_{V} \mathbf{H}(\mathbf{x}) d \mathbf{x}=\sum_{r=1}^{N} f^{(r)}\langle\mathbf{H}\rangle^{(r)},
\end{aligned}
$$

where $f^{(r)}=\left\langle\chi^{(r)}\right\rangle$ is the volume fraction of phase $r$. Under the two kinds of boundary conditions in Eq. (2), the average intensity and flux fields of the RVE are

$$
\langle\mathbf{H}\rangle=\mathbf{H}^{0}=\sum_{r=1}^{N} f^{(r)}\langle\mathbf{H}\rangle^{(r)}, \quad\langle\mathbf{q}\rangle=\mathbf{q}^{0}=\sum_{r=1}^{N} f^{(r)}\langle\mathbf{q}\rangle^{(r)} .
$$

The energy dissipated per unit volume in a linear homogeneous medium is a non-negative quantity proportional to the inner product of the intensity and flux fields, i.e., $W(\mathbf{x})$ $=\frac{1}{2} \mathbf{H}(\mathbf{x}) \cdot \mathbf{q}(\mathbf{x}) \geqslant 0 .{ }^{10}$ It is common to refer to $W(\mathbf{x})$ simply as the "microscopic energy." Equation (1) shows that the microscopic energy is a positive definite quadratic form in either the intensity or the flux, i.e.,

$$
\begin{aligned}
W_{H}(\mathbf{x}, \mathbf{H})= & \frac{1}{2} \mathbf{H}(\mathbf{x}) \cdot \boldsymbol{\mu}(\mathbf{x}) \cdot \mathbf{H}(\mathbf{x}) \\
& \text { or } \\
& W_{q}(\mathbf{x}, \mathbf{q})=\frac{1}{2} \mathbf{q}(\mathbf{x}) \cdot \zeta(\mathbf{x}) \cdot \mathbf{q}(\mathbf{x}) .
\end{aligned}
$$

The effective conduction property of the heterogeneous medium is determined by its effective energy function ${ }^{10}$

$$
\widetilde{W}_{H}\left(\mathbf{H}^{0}\right)=\min _{\mathbf{H} \in A_{U}} \frac{1}{V} \int_{V} W_{H}(\mathbf{x}, \mathbf{H}) d V
$$

or

$$
\widetilde{W}_{q}\left(\mathbf{q}^{0}\right)=\min _{\mathbf{q} \in A_{L}} \frac{1}{V} \int_{V} W_{q}(\mathbf{x}, \mathbf{q}) d V
$$

where $A_{U}$ and $A_{L}$ are trial intensity and flux fields defined by the following sets: ${ }^{10}$

$$
\begin{aligned}
A_{U}= & \left\{\operatorname{ergodic} \mathbf{H}: \boldsymbol{\nabla} \times \mathbf{H}=\mathbf{0},\langle\mathbf{H}\rangle=\mathbf{H}^{0}\right\} \\
& \text { or } \\
& A_{L}=\left\{\operatorname{ergodic} \mathbf{q}: \boldsymbol{\nabla} \cdot \mathbf{q}=0,\langle\mathbf{q}\rangle=\mathbf{q}^{0}\right\} .
\end{aligned}
$$

Because of the linearity, the effective conductivity and resistivity tensors $\overline{\boldsymbol{\mu}}$ and $\overline{\boldsymbol{\zeta}}$ of the heterogeneous medium are defined by the following relations:

$$
\frac{\partial \widetilde{W}_{H}\langle(\mathbf{H})\rangle}{\partial\langle\mathbf{H}\rangle}=\overline{\boldsymbol{\mu}} \cdot\langle\mathbf{H}\rangle \quad \text { or } \quad \frac{\partial \widetilde{W}_{q}(\langle\mathbf{q}\rangle)}{\partial\langle\mathbf{q}\rangle}=\overline{\boldsymbol{\zeta}} \cdot\langle\mathbf{q}\rangle .
$$




\section{EFFECTIVE CONDUCTIVITIES}

Hashin and Shtrikman ${ }^{17}$ introduced a variational procedure to estimate the effective conductivity tensors of heterogeneous media with statistically isotropic microstructures. The Hashin-Shtrikman variational principles are an alterative representation of the effective energy of the heterogeneous medium in terms of suitably chosen polarization fields relative to a homogeneous comparison material for the conduction problem, and rigorous upper and lower bounds for the effective conductivity tensor are obtained by choosing the conductivity of the comparison material to be equal to the maximum and minimum conductivity tensors of the phases, respectively. ${ }^{17} \mathrm{~A}$ generalization of the Hashin-Shtrikman variational principle in various contexts was provided by several authors over the years for the conduction and elasticity problems. ${ }^{22-24}$ For example, Milton and Kohn ${ }^{23}$ obtained the bounds on the effective conductivity of an anisotropic composite consisting of an arbitrary number of possibly anisotropic phases, and the bounds on effective elastic moduli of an anisotropic mixture of two well-ordered isotropic materials. Ponte Castañeda and Willis ${ }^{24}$ proposed an approach for predicting the effective elastic moduli of heterogeneous media, in which the spatial distribution and the shape of the inclusions are separated. In this approach, both the inclusions and their distribution are taken to be ellipsoidal. Thus the effective elastic moduli of the heterogeneous media which are in the form of explicit expressions are dependent on two ellipsoids with independent shapes, one characterizing the shape of the inclusions and the other their distribution function. In the following, we will obtain an estimate of the effective conductivities of heterogeneous media containing multiple inclusions with various spatial distributions and orientations based on the method of Ponte Castañeda and Willis for the elasticity problem. ${ }^{24}$

\section{A. Variational principle based on a homogeneous comparison medium}

Consider an ergodic heterogeneous medium with effective conductivity tensor $\overline{\boldsymbol{\mu}}$ and a homogeneous comparison material (of the same shape and size) with a uniform conductivity tensor $\boldsymbol{\mu}^{(0)}$. Let the local flux polarization field $\boldsymbol{\tau}$ be defined by

$$
\boldsymbol{\tau}=\mathbf{q}-\boldsymbol{\mu}^{(0)} \cdot \mathbf{H}, \quad \mathbf{H} \in A_{U}
$$

Let $W_{H}^{(0)}\left(=\frac{1}{2} \mathbf{H} \cdot \boldsymbol{\mu}^{(0)} \cdot \mathbf{H}\right)$ be the energy associated with the comparison material of uniform conductivity tensor $\boldsymbol{\mu}^{(0)}$, and assume that $\boldsymbol{\mu}^{(0)} \leqslant \min \left\{\boldsymbol{\mu}^{(r)}\right\}$. In this way, $\mathbf{H} \cdot\left(\boldsymbol{\mu}^{(0)}-\boldsymbol{\mu}^{(r)}\right) \cdot \mathbf{H}$ $\leqslant 0$ for all $\mathbf{H} \neq 0(r=1, \ldots, N)$. In analogy with the elasticity problem, ${ }^{25}$ the definition of the Legendre transform for the conduction problem gives

$$
\left(W_{H}-W_{H}^{(0)}\right)^{*}(\mathbf{x}, \boldsymbol{\tau})=\max _{\mathbf{H} \in A_{U}}\left\{\boldsymbol{\tau} \cdot \mathbf{H}-\left[W_{H}(\mathbf{x}, \mathbf{H})-W_{H}^{(0)}(\mathbf{H})\right]\right\} .
$$

From the above equation it follows that

$$
W_{H}(\mathbf{x}, \mathbf{H}) \geqslant W_{H}^{(0)}(\mathbf{H})+\boldsymbol{\tau} \cdot \mathbf{H}-\left(W_{H}-W_{H}^{(0)}\right)^{*}(\mathbf{x}, \boldsymbol{\tau}) .
$$

Substituting Eq. (12) into Eq. (7) gives

$$
\widetilde{W}_{H}\left(\mathbf{H}^{0}\right) \geqslant \min _{\mathbf{H} \in A_{U}}\left\langle W_{H}^{(0)}(\mathbf{H})+\boldsymbol{\tau} \cdot \mathbf{H}-\left(W_{H}-W_{H}^{(0)}\right)^{*}(\mathbf{x}, \boldsymbol{\tau})\right\rangle .
$$

A dual variational principle can be obtained from the second expression of Eq. (7) in terms of intensity polarization tensor. For brevity, we do not reproduce the corresponding procedure and results.

For any chosen polarization field $\boldsymbol{\tau}$ given in Eq. (10), the intensity field $\mathbf{H}$ that attains the minimum of Eq. (13) satisfies $\boldsymbol{\nabla} \cdot\left(\boldsymbol{\mu}^{(0)} \cdot \mathbf{H}\right)=-\boldsymbol{\nabla} \cdot \boldsymbol{\tau}\left(\mathbf{H} \in A_{U}\right)$. Then, making use of the Green's function $\mathbf{G}^{(0)}$ in the domain $V$, it follows that ${ }^{22}$

$$
\mathbf{H}=\mathbf{H}^{0}-\boldsymbol{\Gamma}^{(0)} \boldsymbol{\tau} \quad\left(\mathbf{H} \in A_{U}\right),
$$

where $\boldsymbol{\Gamma}^{(0)}$ is the linear integral operator defined by ${ }^{22}$

$$
\boldsymbol{\Gamma}^{(0)} \boldsymbol{\tau}=\int_{V} \boldsymbol{\Gamma}^{(0)}\left(\mathbf{x}, \mathbf{x}^{\prime}\right) \cdot\left[\boldsymbol{\tau}\left(\mathbf{x}^{\prime}\right)-\langle\boldsymbol{\tau}\rangle\right] d V^{\prime}
$$

whose kernel is related to the Green's function $\mathbf{G}^{(0)}$ in the domain $V$ by

$$
\boldsymbol{\Gamma}_{i j}^{(0)}=G_{, i j}^{(0)}(\mathbf{x})
$$

where $\langle\boldsymbol{\tau}\rangle$ in Eq. (15) denotes the spatial average of $\boldsymbol{\tau}$ over $V$.

Generally, the flux polarization field in Eq. (13) is difficult to determine exactly. It is useful to select the polarization field to be piecewise constant as follows:

$$
\boldsymbol{\tau}(\mathbf{x})=\sum_{r=1}^{N} \chi^{(r)}(\mathbf{x}) \boldsymbol{\tau}^{(r)}
$$

Using $f^{(r)}=\left\langle\chi^{r}\right\rangle$ and the fact that the average of a tensor $\mathbf{B}$ over phase $r$ is given by $\langle\mathbf{B}\rangle^{(r)}=\left\langle\left(\chi^{(r)}(\mathbf{x}) / f^{(r)}\right) \mathbf{B}\right\rangle$, it follows from Eqs. (14) and (15) that $\langle\mathbf{H}\rangle^{(r)}$ is given by

$$
\langle\mathbf{H}\rangle^{(r)}=\mathbf{H}^{0}-\frac{1}{f^{(r)}} \sum_{s=1}^{N} \mathbf{A}^{(r s)} \cdot \boldsymbol{\tau}^{(s)},
$$

where the tensor $\mathbf{A}^{(r s)}$ depends only on the microstructure of the heterogeneous medium and on $\boldsymbol{\mu}^{(0)}$,

$$
\begin{gathered}
\mathbf{A}^{(r s)}=\left\langle\int_{V} \chi^{(r)}(\mathbf{x})\left[\chi^{(s)}\left(\mathbf{x}^{\prime}\right)-f^{(s)}\right] \Gamma^{(0)}\left(\mathbf{x}, \mathbf{x}^{\prime}\right) d V^{\prime}\right\rangle \\
(r, s=1, \ldots, N) .
\end{gathered}
$$

If the distribution of the phases has no long-range order, the finite body kernel in Eq. (15) can be replaced by the corresponding kernel constructed from the infinite body Green's function. ${ }^{22}$ Therefore the kernel now becomes a function of $\left(\mathbf{x}-\mathbf{x}^{\prime}\right)$ only. This replacement is adopted henceforth. Thus, using Eqs. (15) and (17) and the fact that the average of $\boldsymbol{\Gamma}^{(0)} \boldsymbol{\tau}$ is zero [cf. Eq. (14)], Eq. (13) becomes 


$$
\begin{aligned}
\tilde{W}_{H}\left(\mathbf{H}^{0}\right) \geqslant & \frac{1}{2} \mathbf{H}^{0} \cdot \boldsymbol{\mu}^{(0)} \cdot \mathbf{H}^{0}+\langle\boldsymbol{\tau}\rangle \cdot \mathbf{H}^{0} \\
& -\frac{1}{2} \sum_{r=1}^{N} f^{(r)} \boldsymbol{\tau}^{(r)} \cdot\left[\left(\boldsymbol{\mu}^{(r)}-\boldsymbol{\mu}^{(0)}\right)^{-1} \cdot \boldsymbol{\tau}^{(r)}\right] \\
& -\frac{1}{2} \sum_{r=1}^{N} \sum_{s=1}^{N} \boldsymbol{\tau}^{(r)} \cdot \mathbf{A}^{(r s)} \cdot \boldsymbol{\tau}^{(s)}
\end{aligned}
$$

where $\langle\boldsymbol{\tau}\rangle=\sum_{r=1}^{N} f^{(r)} \boldsymbol{\tau}^{(r)}$. The above estimate may be optimized over the polarization fields $\tau^{(r)}(r=1, \ldots, N)$ to give

$$
\widetilde{W}_{H}\left(\mathbf{H}^{0}\right) \geqslant \frac{1}{2} \mathbf{H}^{0} \cdot \boldsymbol{\mu}^{(0)} \cdot \mathbf{H}^{0}+\frac{1}{2}\langle\boldsymbol{\tau}\rangle \cdot \mathbf{H}^{0},
$$

where the optimal flux polarization fields satisfy the following relations:

$$
\left(\boldsymbol{\mu}^{(r)}-\boldsymbol{\mu}^{(0)}\right)^{-1} \cdot \boldsymbol{\tau}^{(r)}+\frac{1}{f^{(r)}} \sum_{s=1}^{N} \mathbf{A}^{(r s)} \cdot \boldsymbol{\tau}^{(s)}=\mathbf{H}^{0} \quad(r=1, \ldots, N)
$$

The lower bound is given by Eq. (21) under the assumption that $\boldsymbol{\mu}^{(0)} \leqslant \min \left\{\boldsymbol{\mu}^{(r)}\right\}$. If, however, it is assumed $\boldsymbol{\mu}^{(0)}$ $\geqslant \max \left\{\boldsymbol{\mu}^{(r)}\right\}$ and the max operation is replaced by a min operation in Eq. (11), then we obtain an upper bound for $W_{H}$ in Eq. (12). Equations (21) and (22) still apply, except that the sign of the inequality must be reversed in Eq. (21) to give an upper bound for $\widetilde{W}_{H}\left(\mathbf{H}^{0}\right)$. However, if neither assumption on $\boldsymbol{\mu}^{(0)}$ is satisfied, the right-hand side of Eq. (21) still provides a variational estimate of $\widetilde{W}_{H}\left(\mathbf{H}^{0}\right)$. In all cases, the estimate of $\widetilde{W}_{H}\left(\mathbf{H}^{0}\right)$ provided by Eqs. (21) and (22) is explicit, except for the microstructural tensor $\mathbf{A}^{(r s)}$, which needs to be determined for the specific class of microstructure. In the following, we will give an estimate of the effective conductivity for ergodic heterogeneous media with prescribed twopoint correlation functions for the statistics of the distribution of centers of ellipsoidal inclusions.

\section{B. Estimates of effective conductivities}

For an ergodic heterogeneous medium consisting of inclusions of $N-1$ different types in a homogeneous matrix, the matrix will be denoted by the superscript 1 , and its conductivity tensor and volume fraction by $\boldsymbol{\mu}^{(1)}$ and $f^{(1)}$, respectively. The inclusions with conductivity tensor $\boldsymbol{\mu}^{(r)}$ and the volume fraction $f^{(r)}$ may differ in size, shape and orientation. Let the RVE with volume $V$ occupy the region of space $\Omega$. Assume that there are $n^{(r)}(r=2, \ldots, N-1)$ inclusions of type $r$, the $i$ th inclusion of type $r$ occupies the domain $\Omega_{i}^{(r)}$ in $\Omega$, and its center is at the random point $\mathbf{x}_{i}^{(r)}\left(i=1, \ldots, n^{(r)}\right)$. Let $\chi_{i}^{(r)}(\mathbf{x})$ be the characteristic function of the reference inclusion $\Omega_{i}^{(r)}\left[\chi_{i}^{(r)}(\mathbf{x})=1\right.$ if $\mathbf{x} \in \Omega_{i}^{(r)}$ and 0 otherwise $]$. It follows that the characteristic function of phase $r$ is ${ }^{24}$

$$
\chi^{(r)}(\mathbf{x})=\int_{\Omega}\left[\chi_{i}^{(r)}(\mathbf{x}-\mathbf{z}) \Psi^{(r)}(\mathbf{z})\right] d z
$$

where $\Psi^{(r)}(\mathbf{z})=\sum_{i=1}^{n^{(r)}} \delta\left(\mathbf{z}-\mathbf{x}_{i}^{(r)}\right)$. Define the two-point correlation function of finding simultaneously phase $r$ at $\mathbf{x}$ and phase $s$ at $\mathbf{x}^{\prime}$ as

$$
p^{(r s)}\left(\mathbf{x}, \mathbf{x}^{\prime}\right)=\left\langle\chi^{(r)}(\mathbf{x}) \chi^{(s)}\left(\mathbf{x}^{\prime}\right)\right\rangle
$$

where statistical homogeneity has been assumed, so that $p^{(r s)}\left(\mathbf{x}, \mathbf{x}^{\prime}\right)=p^{(r s)}\left(\mathbf{x}-\mathbf{x}^{\prime}\right)$. It should be mentioned that $\left\langle\mathbf{A}^{(r s)}\right\rangle$ is symmetric in the superscripts $r$ and $s$ due to the assumption of statistical homogeneity and the fact that $\Gamma^{(0)}(\mathbf{x})=\Gamma^{(0)}$ $(-\mathbf{x})$. Moreover, the symmetry of $\left\langle\mathbf{A}^{(r s)}\right\rangle$ ensures corresponding symmetry of $\overline{\boldsymbol{\mu}}$.

In particular, for $N$-phase heterogeneous media consisting of $N-1$ types of ellipsoidal inclusions, distributed with ellipsoidal symmetry in the matrix (i.e., phase 1), the microstructural tensor $\mathbf{A}^{(r s)}$ in Eq. (19) can be simplified. Analogous to the work of Ponte Castañeda and Willis ${ }^{24}$ for the elasticity problem, assume that the two-point correlation function has ellipsoidal symmetry, i.e., $p^{(r s)}(\mathbf{x})=p^{(r s)}\left(\left|\mathbf{Z}_{d}^{(r s)} \cdot \mathbf{x}\right|\right)$ for some constant, symmetric tensor $\mathbf{Z}_{d}^{(r s)}$, which defines a distribution ellipsoid, $\Omega_{d}^{(r s)}=\left\{\mathbf{x}:\left|\mathbf{Z}_{d}^{(r s)} \cdot \mathbf{x}\right|^{2}<1\right\}$. The symmetry requirement means $\mathbf{Z}_{d}^{(r s)}$ should be symmetric in the superscripts $r$ and $s$, so that $\Omega_{d}^{(r s)}=\Omega_{d}^{(s r)}$. Furthermore, it is assumed that the phases do not overlap. Likewise, if the inclusions are assumed to be of ellipsoidal shape, i.e., $\Omega_{i}^{(r)}=\left\{\mathbf{x}:\left|\mathbf{Z}_{i}^{(r)} \cdot \mathbf{x}\right|^{2}<1\right\}$, $\left(r=2, \ldots, N ; i=1, \ldots, n^{(r)}\right)$ then $\left\langle\mathbf{A}^{(r s)}\right\rangle$ for this kind of heterogeneous medium can be expressed as

$$
\left\langle\mathbf{A}^{(r s)}\right\rangle=f^{(r)}\left(\delta_{r s} \mathbf{E}_{i}^{(r)}-f^{(s)} \mathbf{E}_{d}^{(r s)}\right) \quad(r, s=2, \ldots, N),
$$

where tensors $\mathbf{E}_{i}^{(r)}$ and $\mathbf{E}_{d}^{(r s)}$ are associated with the inclusion and distribution shape tensors $\mathbf{Z}_{i}^{(r)}$ and $\mathbf{Z}_{d}^{(r s)}$, respectively, and $\mathbf{E}_{i}^{(r)}$ can be expressed as ${ }^{22}$

$$
\mathbf{E}_{i}^{(r)}=\frac{1}{4 \pi\left|\mathbf{Z}_{i}^{(r)}\right|} \int_{|\boldsymbol{\xi}|=1} \mathbf{C}^{(0)}(\boldsymbol{\xi})\left|\left(\mathbf{Z}_{i}^{(r)}\right)^{-1} \cdot \boldsymbol{\xi}\right|^{-3} d S(\boldsymbol{\xi}),
$$

where the integration is performed over the unit sphere $|\xi|$ $=1, C_{m n}^{(0)}(\boldsymbol{\xi})=F \xi_{m} \xi_{n}$, and $F=1 / \mu_{k l}^{(0)} \xi_{k} \xi_{l}$, and $\xi_{p}$ is the component of the unit vector. $\mathbf{E}_{d}^{(r s)}$ is given by an expression analogous to Eq. (26) with $\mathbf{Z}_{i}^{(r)}$ replaced by $\mathbf{Z}_{d}^{(r s)}$. It is noted that the inverses of the eigenvalues of $\mathbf{Z}_{i}^{(s)}$ and $\mathbf{Z}_{d}^{(r s)}$ are the semiaxes of the ellipsoidal inclusion and the distribution ellipsoid, respectively. Ponte Castañeda and Willis ${ }^{24}$ obtained an expression similar to Eq. (25) for the elasticity problem. Details of the procedure for obtaining Eq. (25) can be found in the work of Ponte Castañeda and Willis. ${ }^{24}$

For the ergodic heterogeneous medium under consideration, a good choice for the comparison material is the matrix material itself $\left(\boldsymbol{\mu}^{(0)}=\boldsymbol{\mu}^{(1)}\right)$, so that the polarization field vanishes exactly in the matrix phase. In this case, Eqs. (22) and (25) lead to 


$$
\begin{aligned}
& {\left[\left(\boldsymbol{\mu}^{(r)}-\boldsymbol{\mu}^{(1)}\right)^{-1}+\mathbf{E}_{i}^{(r)}\right] \cdot \boldsymbol{\tau}^{(r)}-\sum_{s=2}^{N} f^{(s)} \mathbf{E}_{d}^{(r s)} \cdot \boldsymbol{\tau}^{(s)}=\mathbf{H}^{0}} \\
& (r=2, \ldots, N) .
\end{aligned}
$$

Substituting $\tau^{(r)}(r=2, \ldots, N)$ from Eq. (27) into Eq. (21) gives an estimate of the effective conductivity tensor $\bar{\mu}$. Such an estimate will be a lower bound, if the matrix happens to be the lowest conducting phase in the heterogeneous medium, and an upper bound, if the matrix is the highest conducting phase. On the other hand, if the matrix is neither the lowest nor the highest conducting phase, the estimate will be neither an upper bound nor a lower bound, but will be simply an estimate of $\overline{\boldsymbol{\mu}}$.

In particular, when the distribution of the inclusions is taken to be the same for all inclusion pairs, i.e., $\mathbf{E}_{d}^{(r s)}=\mathbf{E}_{d}$, for $r, s=2, \ldots, N$, relatively simple expressions are obtained for the effective conductivity $\overline{\boldsymbol{\mu}}$. In the following, $\mathbf{E}_{d}$ and $\mathbf{E}_{i}^{(r)}$ will be expressed by the geometrical factor tensors $\mathbf{S}^{(V)}$ and $\mathbf{S}^{(r)}$ of the distribution ellipsoid and the $r$ th ellipsoidal inclusion, respectively. Tensors $\mathbf{S}^{(V)}$ and $\mathbf{S}^{(r)}$ are analogous to the Eshelby tensor for elasticity, ${ }^{26}$ and we can obtain $\mathbf{S}^{(V)}$ and $\mathbf{S}^{(r)}$ for the anisotropic medium following the works of Willis, ${ }^{22}$ and Hatta and Taya. ${ }^{27}$ When an ellipsoidal domain with uniform eigenintensity field $\mathbf{H}^{*}$ exists in an infinite matrix, the flux field $\mathbf{q}$ is expressed as $\mathbf{q}=\boldsymbol{\mu}^{(1)} \cdot\left(\mathbf{H}-\mathbf{H}^{*}\right)$, where $\mathbf{H}$ is the intensity field caused by $\mathbf{H}^{*}$. For steady state conduction $(\boldsymbol{\nabla} \cdot \mathbf{q}=0)$, the differential equation $\boldsymbol{\nabla} \cdot\left[\boldsymbol{\mu}^{(1)} \cdot(\mathbf{H}\right.$ $\left.\left.-\mathbf{H}^{*}\right)\right]=0$ can be obtained. By using a mathematical treatment similar to that used for deriving the fourth-order Eshelby tensor for elastically anisotropic medium, ${ }^{28}$ the second-order tensor $\mathbf{S}^{(r)}$ can be obtained as

$$
\mathbf{S}^{(r)}=\frac{a_{1}^{(r)} a_{2}^{(r)} a_{3}^{(r)}}{4 \pi} \int_{|\xi|=1} \frac{\mathbf{C}^{(0)}(\boldsymbol{\xi}) \cdot \boldsymbol{\mu}^{(1)}}{\Upsilon^{3}} d S(\boldsymbol{\xi})
$$

where $Y^{2}=\left(a_{1}^{(r)}\right)^{2} \dot{\xi}_{1}^{2}+\left(a_{2}^{(r)}\right)^{2} \dot{\xi}_{2}^{2}+\left(a_{3}^{(r)}\right)^{2} \xi_{3}^{2}$ and $a_{1}^{(r)}, a_{2}^{(r)}$, and $a_{3}^{(r)}$ denote the semi-axes of the $r$ th ellipsoidal inclusion. $\mathbf{S}^{(V)}$ is given by an expression analogous to Eq. (28) with $a_{i}^{(r)}(i$ $=1,2,3)$ replaced by $a_{i}^{(V)} . \mathbf{S}^{(V)}$ and $\mathbf{S}^{(r)}$ for various ellipsoids are given in the Appendix. From Eqs. (26) and (28), the relations between $\mathbf{E}_{d}$ and $\mathbf{S}^{(V)}, \mathbf{E}_{i}^{(r)}$ and $\mathbf{S}^{(r)}$, can be obtained as

$$
\mathbf{E}_{d}=\mathbf{S}^{(V)} \cdot\left(\boldsymbol{\mu}^{(1)}\right)^{-1}, \quad \mathbf{E}_{i}^{(r)}=\mathbf{S}^{(r)} \cdot\left(\boldsymbol{\mu}^{(1)}\right)^{-1} .
$$

Therefore, the effective conductivity $\overline{\boldsymbol{\mu}}$ for an $N$-phase heterogeneous medium consisting of $N-1$ types of ellipsoidal inclusions, distributed with ellipsoidal symmetry in the matrix (phase 1), can be obtained from Eqs. (21), (27), and (29),

$$
\overline{\boldsymbol{\mu}}=\boldsymbol{\mu}^{(1)}+\boldsymbol{\mu}^{(1)} \cdot\left[\left(\sum_{r=2}^{N} \mathbf{T}^{(r)}\right)^{-1}-\mathbf{S}^{(V)}\right]^{-1}
$$

in which

$$
\mathbf{T}^{(r)}=f^{(r)}\left[\left(\boldsymbol{\mu}^{(r)}-\boldsymbol{\mu}^{(1)}\right)^{-1} \cdot \boldsymbol{\mu}^{(1)}+\mathbf{S}^{(r)}\right]^{-1} .
$$

Equations (30) and (31) are the basic equations for predicting the effective conductivities of the heterogeneous medium. These equations are the counterparts of Eqs. (3.20) and
(3.21) in the paper of Ponte Castañeda and Willis for elasticity problems. ${ }^{24}$

If all ellipsoidal inclusions have the same shape and are aligned in the same direction, and the shape and orientation of the distribution ellipsoid are identical to those of the ellipsoidal inclusions, i.e., $\mathbf{S}^{(V)}=\mathbf{S}^{(r)}$, then Eqs. (30) and (31) reduce to the results given by the Mori-Tanaka method (MTM) ${ }^{29}$ If the inclusions and the distribution ellipsoid are spherical in shape, Eqs. (30) and (31) reduce to the results given by the Mori-Tanaka method for a heterogeneous medium containing randomly distributed spherical inclusions. ${ }^{29}$

To explore the present scheme further, the effective conductivity tensor in Eq. (30) is expanded in terms of the volume fraction of the inclusions

$$
\begin{aligned}
\overline{\boldsymbol{\mu}}= & \boldsymbol{\mu}^{(1)}+\boldsymbol{\mu}^{(1)} \cdot\left[\sum_{r=2}^{N} \mathbf{T}^{(r)}+\left(\sum_{r=2}^{N} \mathbf{T}^{(r)}\right) \cdot \mathbf{S}^{(V)} \cdot\left(\sum_{r=2}^{N} \mathbf{T}^{(r)}\right)\right] \\
& +O\left[\left(f^{(r)}\right)^{3}\right] .
\end{aligned}
$$

Equation (32), together with Eq. (31), shows that the shape of the inclusions affects $\overline{\boldsymbol{\mu}}$ through the tensor $\mathbf{S}^{(r)}$ to the first order in the volume fraction of the inclusions, whereas the corresponding shape of the distribution ellipsoid is seen to affect $\overline{\boldsymbol{\mu}}$ through $\mathbf{S}^{(V)}$ to the second order in the volume fraction of the inclusions. If the terms higher than the first order in the volume fraction of the inclusions are ignored, Eq. (32) reduces to the results of Shafiro and Kachanov. ${ }^{16}$ Equation (32), with the first and the second terms retained on the righthand side, can therefore be regarded as the generalization of the Maxwell formula to media containing multiple inclusions, which takes into account the effect of the orientation of the inclusions. Detailed discussion is given below in Sec. IV.

It should be noted that for consistency with the assumption of impenetrability of the inclusions, the volume fraction of the inclusions should be in a safe range at given aspect ratios of the ellipsoidal inclusions. Ponte Castañeda and Willis. ${ }^{24}$ have stated this for the elasticity problem, and this restriction on the volume fraction is also applicable to the present conduction problem. Furthermore, when the spatial correlations of inclusion locations are of ellipsoidal forms, the shape of the inclusion is independent of that of the distribution ellipsoid.

\section{HETEROGENEOUS MEDIA WITH MULTIPLE INCLUSIONS}

\section{A. Aligned ellipsoidal inclusions}

If a heterogeneous medium contains aligned ellipsoidal inclusions whose semi-axes are parallel to the axes of $x, y$, and $z$ of the global reference frame, respectively, and the shape of the distribution is also ellipsoidal, then the effective principal conductivities of the medium are obtained from Eqs. (30) and (31), i.e.,

$$
\bar{\mu}_{j}=\mu^{(1)}+\mu^{(1)}\left[\left(\sum_{r=2}^{N} f^{(r)} A_{j}^{(r)} \frac{\mu^{(r)}-\mu^{(1)}}{\mu^{(1)}}\right)^{-1}-S_{j j}^{(V)}\right]^{-1}
$$

(no sum over $j$ ),

where $j=x, y, z, S_{j j}^{(V)}$ is given by Eq. (A1), and $A_{j}^{(r)}$ are given by 
$A_{j}^{(r)}=\left(1+S_{j j}^{(r)} \frac{\left(\mu^{(r)}-\mu^{(1)}\right)}{\mu^{(1)}}\right)^{-1}, j=x, y, z \quad$ (no sum over $\left.j\right)$.

In particular, if a heterogeneous medium contains aligned spheroidal inclusions and the inclusions also have a spheroidal distribution aligned with the inclusions, the effective conductivity property will be transversely isotropic. In this case, define shape parameters $g^{(r)}\left(g^{(r)}=2 S_{11}^{(r)}=2 S_{22}^{(r)}\right)$ and $g^{(V)}\left(g^{(V)}=2 S_{11}^{(V)}=2 S_{22}^{(V)}\right)$ for the spheroidal inclusion and the distribution spheroid. Then, the transverse conductivity $\bar{\mu}_{T}$ of the heterogeneous medium is

$\bar{\mu}_{T}=\mu^{(1)}+\mu^{(1)}\left\{\left[\sum_{r=2}^{N} f^{(r)}\left(\frac{\mu^{(1)}}{\mu^{(r)}-\mu^{(1)}}+\frac{g^{(r)}}{2}\right)^{-1}\right]^{-1}-\frac{g^{(V)}}{2}\right\}^{-1}$

and the longitudinal conductivity $\bar{\mu}_{L}$ is

$$
\begin{aligned}
\bar{\mu}_{L}= & \mu^{(1)}+\mu^{(1)}\left\{\left[\sum_{r=2}^{N} f^{(r)}\left(\frac{\mu^{(1)}}{\mu^{(r)}-\mu^{(1)}}+1-g^{(r)}\right)^{-1}\right]^{-1}\right. \\
& \left.-1+g^{(V)}\right\}^{-1} .
\end{aligned}
$$

For insulating inclusions $\left(\mu^{(r)}=0\right)$, Eqs. (35) and (36) reduce to

$$
\begin{gathered}
\bar{\mu}_{T}=\mu^{(1)}-\mu^{(1)}\left\{\left[\sum_{r=2}^{N} f^{(r)}\left(1-\frac{g^{(r)}}{2}\right)^{-1}\right]^{-1}+\frac{g^{(V)}}{2}\right\}^{-1}, \\
\bar{\mu}_{L}=\mu^{(1)}-\mu^{(1)}\left[\left(\sum_{r=2}^{N} \frac{f^{(r)}}{g^{(r)}}\right)^{-1}+1-g^{(V)}\right]^{-1},
\end{gathered}
$$

and for superconducting inclusions $\left(\mu^{(r)} \rightarrow \infty\right)$,

$$
\begin{gathered}
\bar{\mu}_{T}=\mu^{(1)}+\mu^{(1)}\left[\left(\sum_{r=2}^{N} \frac{2 f^{(r)}}{g^{(r)}}\right)^{-1}-\frac{g^{(V)}}{2}\right]^{-1}, \\
\bar{\mu}_{L}=\mu^{(1)}+\mu^{(1)}\left[\left(\sum_{r=2}^{N} \frac{f^{(r)}}{1-g^{(r)}}\right)^{-1}-1+g^{(V)}\right]^{-1} .
\end{gathered}
$$

For a heterogeneous medium containing aligned circular cracks $\left(a_{3}^{(r)} \rightarrow 0, \lambda^{(r)}=1\right)$, it follows from Eq. (31),

$$
\sum_{r=2}^{N} \mathbf{T}^{(r)}=-\frac{8}{3 V} \sum_{r=2}^{N}\left[\left(a^{(r)}\right)^{3} \mathbf{e}_{3} \otimes \mathbf{e}_{3}\right]=-\frac{8}{3} \boldsymbol{\alpha},
$$

where $\alpha=(1 / V) \sum_{r=2}^{N}\left[\left(a^{(r)}\right)^{3} \mathbf{e}_{3} \otimes \mathbf{e}_{3}\right]$ is the symmetric crack density tensor of the second order for the circular cracks, ${ }^{16}$ and $\mathbf{e}_{3}$ is the unit vector normal to the cracks. Therefore, the effective conductivities of the heterogeneous medium containing aligned circular cracks are

$$
\bar{\mu}_{T}=\mu^{(1)}, \quad \bar{\mu}_{L}=\mu^{(1)}-\mu^{(1)}\left[\left(\frac{8 \rho}{3}\right)^{-1}+1-g^{(V)}\right]^{-1}
$$

in which $\rho=\operatorname{tr} \boldsymbol{\alpha}=(1 / V) \sum_{r=2}^{N}\left(a^{(r)}\right)^{3}$ is the scalar crack density. ${ }^{16}$

It is seen from Eqs. (32) and (33) that at low volume fractions, when the terms higher than the second order in the volume fraction $f^{(r)}$ of the inclusions can be ignored, the effective principal conductivities of heterogeneous media containing multiple aligned ellipsoidal inclusions can be expressed as

$$
\begin{aligned}
\bar{\mu}_{j}= & \mu^{(1)}+\sum_{r=2}^{N} f^{(r)} A_{j}^{(r)}\left(\mu^{(r)}-\mu^{(1)}\right) \\
& \left.+\mu^{(1)}\left(\sum_{r=2}^{N} f^{(r)} A_{j}^{(r)} \frac{\mu^{(r)}-\mu^{(1)}}{\mu^{(1)}}\right)^{2} S_{j j}^{(V)} \quad \text { (no sum over } j\right),
\end{aligned}
$$

where $j=x, y, z$, and $A_{j}^{(r)}$ is given by Eq. (34). If the terms higher than the first order in the volume fraction of the inclusions are ignored, Eq. (43) reduces to Eqs. (2) and (14) in the paper of Shafiro and Kachanov. ${ }^{16}$

For a three-phase heterogeneous medium containing aligned ellipsoidal inclusions (with volume fraction $f^{(2)}$ ) and spherical inclusions (with volume fraction $f^{(3)}$ ), Eq. (43) becomes

$$
\begin{aligned}
\bar{\mu}_{j}= & \mu^{(1)}+f^{(2)}\left(\mu^{(2)}-\mu^{(1)}\right) A_{j}^{(2)}+\frac{3 f^{(3)} \mu^{(1)}}{2 \mu^{(1)}+\mu^{(3)}}\left(\mu^{(3)}-\mu^{(1)}\right) \\
& +\left[f^{(2)}\left(\mu^{(2)}-\mu^{(1)}\right) A_{j}^{(2)}+\frac{3 f^{(3)} \mu^{(1)}}{2 \mu^{(1)}+\mu^{(3)}}\left(\mu^{(3)}-\mu^{(1)}\right)\right] \frac{S_{j j}^{(V)}}{\mu^{(1)}}
\end{aligned}
$$

(no sum over $j$ ),

where $A_{j}^{(2)}$ is given by Eq. (34), and the superscript " 2 " denotes a quantity related to the ellipsoidal inclusions. For a two-phase heterogeneous medium containing aligned ellipsoidal inclusions (with volume fraction $f^{(2)}$ ), Eq. (44) with only the first and the second terms retained on the right-hand side reduces to Eq. (10) in the paper of Giordano, ${ }^{14}$ i.e., to the Maxwell formula for aligned ellipsoids with the same shape and conductivity. Therefore, Eq. (43) with the first and the second terms retained on the right-hand side can be regarded as the generalized Maxwell formula for aligned multiple ellipsoids.

\section{B. Randomly oriented and distributed ellipsoidal inclusions}

A heterogeneous medium containing randomly oriented and distributed ellipsoids is isotropic. Therefore, the shape of the distribution ellipsoid should be spherical. In this case, $\mathbf{T}^{(r)}$ in Eq. (31) can be expressed as

$$
\mathbf{T}^{(r)}=h^{(r)} \mathbf{I}
$$

in which $\mathbf{I}$ is the second order identity tensor in threedimensional space, and $h^{(r)}$ is 


$$
h^{(r)}=\frac{1}{3} f^{(r)}\left(\frac{\mu^{(r)}}{\mu^{(1)}}-1\right)\left(A_{1}^{(r)}+A_{2}^{(r)}+A_{3}^{(r)}\right),
$$

where $A_{j}^{(r)}(j=1,2,3)$ is given by Eq. (34). Therefore, the isotropic effective conductivity in Eq. (30) is given by

$$
\bar{\mu}=\mu^{(1)}+\mu^{(1)}\left[\left(\sum_{r=2}^{N} h^{(r)}\right)^{-1}-\frac{1}{3}\right]^{-1} .
$$

For randomly oriented spheroids of general aspect ratio $\gamma^{(r)}$, $h^{(r)}$ in Eq. (47) can be expressed as

$$
\begin{aligned}
h^{(r)}= & \frac{1}{3} f^{(r)}\left[2\left(\frac{\mu^{(1)}}{\mu^{(r)}-\mu^{(1)}}+\frac{g^{(r)}}{2}\right)^{-1}\right. \\
& \left.+\left(\frac{\mu^{(1)}}{\mu^{(r)}-\mu^{(1)}}+1-g^{(r)}\right)^{-1}\right] .
\end{aligned}
$$

For insulating spheroidal inclusions $\left(\mu^{(r)}=0\right)$, Eq. (48) reduces to

$$
h^{(r)}=-\frac{1}{3} f^{(r)}\left(\frac{4}{2-g^{(r)}}+\frac{1}{g^{(r)}}\right),
$$

and for superconducting spheroidal inclusions $\left(\mu^{(r)} \rightarrow \infty\right)$

$$
h^{(r)}=\frac{1}{3} f^{(r)}\left(\frac{4}{g^{(r)}}+\frac{1}{1-g^{(r)}}\right) \text {. }
$$

The isotropic effective conductivity for spherical inclusions without limit on the conductivity contrast between the inclusions and the matrix is

$$
\bar{\mu}=\mu^{(1)}+\mu^{(1)}\left[\left(\sum_{r=2}^{N} 3 f^{(r)} \frac{\mu^{(r)}-\mu^{(1)}}{\mu^{(r)}+2 \mu^{(1)}}\right)^{-1}-\frac{1}{3}\right]^{-1} .
$$

In the case of insulating spherical inclusions,

$$
\bar{\mu}=\mu^{(1)}-\mu^{(1)}\left[\left(\frac{3}{2} \sum_{r=2}^{N} f^{(r)}\right)^{-1}+\frac{1}{3}\right]^{-1},
$$

and in the case of superconducting spherical inclusions,

$$
\bar{\mu}=\mu^{(1)}+\mu^{(1)}\left[\left(3 \sum_{r=2}^{N} f^{(r)}\right)^{-1}-\frac{1}{3}\right]^{-1} .
$$

For randomly oriented circular cracks, the isotropic effective conductivity is expressed in terms of the usual crack density parameter $\rho$ as

$$
\bar{\mu}=\mu^{(1)}-\mu^{(1)}\left[\left(\frac{8}{9} \rho\right)^{-1}+\frac{1}{3}\right]^{-1} .
$$

It can be seen from Eqs. (32) and (47) that if the terms higher than the second order in the volume fraction of the inclusions are ignored, the effective conductivities of heterogeneous media containing multiple randomly oriented and distributed ellipsoidal inclusions can be expressed as

$$
\bar{\mu}=\mu^{(1)}+\mu^{(1)} \sum_{r=2}^{N} h^{(r)}+\frac{\mu^{(1)}}{3}\left(\sum_{r=2}^{N} h^{(r)}\right)^{2} .
$$

If the terms higher than the first order in the volume fraction of the inclusions are ignored, Eq. (55) reduces to the corresponding formula in paper of Shafiro and Kachanov. ${ }^{16}$

For a three-phase heterogeneous medium containing randomly oriented ellipsoidal inclusions (with volume fraction $f^{(2)}$ ) and spherical inclusions (with volume fraction $f^{(3)}$ ), Eq. (55) reduces to

$$
\begin{aligned}
\bar{\mu}= & \mu^{(1)}+\mu^{(1)}\left(h^{(2)}+3 f^{(3)} \frac{\mu^{(3)}-\mu^{(1)}}{2 \mu^{(1)}+\mu^{(3)}}\right) \\
& +\frac{\mu^{(1)}}{3}\left(h^{(2)}+3 f^{(3)} \frac{\mu^{(3)}-\mu^{(1)}}{2 \mu^{(1)}+\mu^{(3)}}\right)^{2},
\end{aligned}
$$

where $h^{(2)}$ is given by Eq. (46), and the superscript " 2 " denotes a quantity related to the ellipsoidal inclusions. For a two-phase heterogeneous medium containing randomly oriented ellipsoidal inclusions (with volume fraction $f^{(2)}$ ), Eq. (56) with only the first and the second terms retained on the right-hand side reduces to Eq. (25) in the paper of Giordano, ${ }^{14}$ i.e., to the Maxwell formula for randomly oriented ellipsoids with the same shape and conductivity. Therefore, Eq. (55) with only the first and the second terms retained on the right-hand side can be regarded as the generalized Maxwell formula for randomly oriented and distributed multiple ellipsoids.

\section{Inclusions with orientational distributions}

For simplicity, we consider a dispersion of spheroidal inclusions embedded in a homogeneous matrix and focus on the analysis of the effect of their orientational distribution on the effective conductivity of the heterogeneous medium. The heterogeneous medium is subjected to a uniform intensity field $\mathbf{H}^{0}\left(H_{0 x}, H_{0 y}, H_{0 z}\right)$. Now consider a single spheroidal inclusion in the heterogeneous medium. The local coordinate system $\left(x_{1}, y_{1}, z_{1}\right)$ is used to describe this spheroidal inclusion with the axis $z_{1}$ directed along its axis of symmetry. Without loss of generality, we let the local axis $y_{1}$ lie in the global $x y$ plane; thus the orientation of this spheroid is defined by angles $\theta$ and $\varphi$ as shown in Fig. 1. The three semiaxes $a_{1}, a_{2}$, and $a_{3}$ are aligned with the coordinate axes $x_{1}$, $y_{1}$, and $z_{1} . \mathbf{e}_{1}, \mathbf{e}_{2}$, and $\mathbf{e}_{3}$ are the unit vectors along the three semi-axes of the spheroid, and $\mathbf{e}_{x}, \mathbf{e}_{y}$, and $\mathbf{e}_{z}$ are the unit base vectors of the global coordinate system $(x, y, z)$ (Fig. 1). Thus, $\mathbf{e}_{3}$ along the axis of symmetry of the $r$ th spheriod in terms of the two angles $\theta \in[0, \pi / 2]$ and $\varphi \in[0,2 \pi]$ (Fig. 1) can be expressed as

$$
\mathbf{e}_{3}=\sin \theta \cos \varphi \mathbf{e}_{x}+\sin \theta \sin \varphi \mathbf{e}_{y}+\cos \theta \mathbf{e}_{z} .
$$

For convenience, $\mathbf{T}^{(r)}$ in Eq. (31) for a spheroidal inclusion is rewritten as

$$
\mathbf{T}^{(r)}=k_{1}^{(r)} \mathbf{I}+k_{2}^{(r)} \mathbf{e}_{3} \otimes \mathbf{e}_{3}
$$

in which 


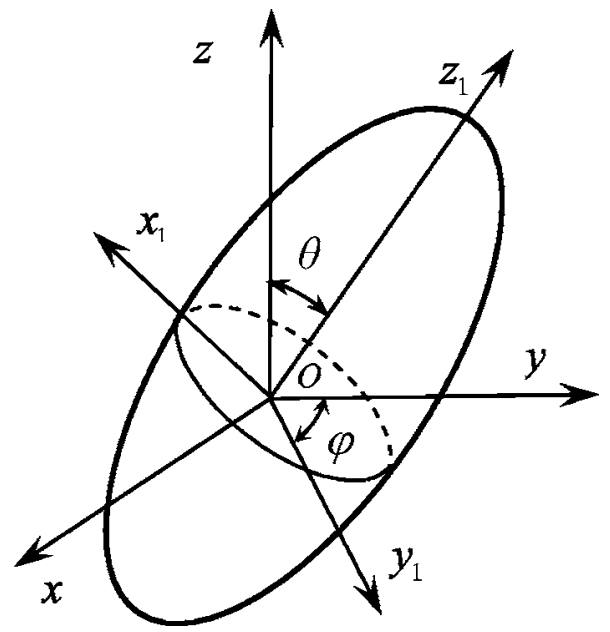

FIG. 1. Transformation between local coordinate system $\left(x_{1}, y_{1}, z_{1}\right)$ and global coordinate system $(x, y, z)$.

$$
\begin{gathered}
k_{1}^{(r)}=f^{(r)}\left(\frac{\mu^{(1)}}{\mu^{(r)}-\mu^{(1)}}+\frac{g^{(r)}}{2}\right)^{-1}, \\
k_{2}^{(r)}=f^{(r)}\left[\left(\frac{\mu^{(1)}}{\mu^{(r)}-\mu^{(1)}}+1-g^{(r)}\right)^{-1}-\left(\frac{\mu^{(1)}}{\mu^{(r)}-\mu^{(1)}}+\frac{g^{(r)}}{2}\right)^{-1}\right] .
\end{gathered}
$$

Introduce a probability density function $D(\theta, \varphi)$ of the inclusion orientations, which is subjected to the condition $\int_{0}^{2 \pi} \int_{0}^{\pi / 2} D(\theta, \varphi) \sin \theta d \theta d \varphi=1$. Assume that there is no correlation between the shape and orientation of the ellipsoidal inclusions (i.e., the RVE contains a sufficiently large number of inclusions). Then the following expression can be obtained from Eq. (58):

$$
\sum_{r=2}^{N} \mathbf{T}^{(r)}=\left\{k_{1}^{(r)}\right\} \mathbf{I}+\left\{k_{2}^{(r)}\right\} \int_{0}^{2 \pi} \int_{0}^{\pi / 2} \mathbf{e}_{3} \otimes \mathbf{e}_{3} D(\theta, \varphi) \sin \theta d \theta d \varphi
$$

in which

$$
\left\{k_{1}^{(r)}\right\}=\sum_{r=2}^{N} k_{1}^{(r)}, \quad\left\{k_{2}^{(r)}\right\}=\sum_{r=2}^{N} k_{2}^{(r)} .
$$

$D(\theta, \varphi)=\delta(\theta)$ (where $\delta$ is the Dirac function) corresponds to aligned inclusions. $D(\theta, \varphi)=$ const corresponds to randomly oriented inclusions. We now consider several orientational distributions by specifying the probability density $D(\theta, \varphi)$ $\equiv D(\theta)$ that does not depend on angle $\varphi$ and assume that the inclusions have a spheroidal distribution aligned with the global $z$ axis. Then the heterogeneous medium is transversely isotropic and Eq. (61) can be expressed in the following form:

$$
\sum_{r=2}^{N} \mathbf{T}^{(r)}=\left\{k_{1}^{(r)}\right\} \mathbf{I}+\left\{k_{2}^{(r)}\right\}\left[R_{1}\left(\mathbf{e}_{x} \otimes \mathbf{e}_{x}+\mathbf{e}_{y} \otimes \mathbf{e}_{y}\right)+R_{2} \mathbf{e}_{z} \otimes \mathbf{e}_{z}\right]
$$

where $R_{1}$ and $R_{2}$ are two constants related to the probability density function $D(\theta)$. Thus, the transversely isotropic effective conductivities for this kind of distribution $[D(\theta, \varphi)$ $\equiv D(\theta)]$ can be obtained from Eqs. (30) and (63), i.e.,

$$
\begin{gathered}
\bar{\mu}_{T}=\mu^{(1)}+\mu^{(1)}\left[\left(\left\{k_{1}^{(r)}\right\}+R_{1}\left\{k_{2}^{(r)}\right\}\right)^{-1}-\frac{g^{(V)}}{2}\right]^{-1}, \\
\bar{\mu}_{L}=\mu^{(1)}+\mu^{(1)}\left[\left(\left\{k_{1}^{(r)}\right\}+R_{2}\left\{k_{2}^{(r)}\right\}\right)^{-1}-1+g^{(V)}\right]^{-1} .
\end{gathered}
$$

If $D(\theta, \varphi)$ in Eq. (61) has the cosine form, i.e., $D(\theta, \varphi)$ $=\cos \theta$, then $R_{1}=1 / 4, R_{2}=1 / 2$. If $D(\theta, \varphi)$ in Eq. (61) has the sine form, i.e., $D(\theta, \varphi)=\sin \theta$, then, $R_{1}=3 / 8, R_{2}=1 / 4$.

Consider an orientational distribution that is intermediate between the random and the aligned ones, for example, the probability density function $D(\theta, \varphi),{ }^{16}$

$$
D(\theta, \varphi)=\frac{1}{2 \pi}\left[\left(\Lambda^{2}+1\right) e^{-\Lambda \theta}+\Lambda e^{-\Lambda \pi / 2}\right] .
$$

The orientational distribution density $D(\theta, \varphi)$ in Eq. (66) can cover several orientations: (a) the random orientation, which corresponds to $\Lambda=0$; (b) the aligned orientation, which corresponds to $\Lambda=\infty$; (c) the slightly-perturbed aligned orientations; and (d) the weakly expressed orientational preference. Equations (61), (63), and (66) lead to

$$
R_{1}=\frac{18-\Lambda\left(\Lambda^{2}+3\right) e^{-\Lambda \pi / 2}}{6\left(\Lambda^{2}+9\right)}, \quad R_{2}=\frac{\left(\Lambda^{2}+3\right)\left(3+\Lambda e^{-\Lambda \pi / 2}\right)}{3\left(\Lambda^{2}+9\right)}
$$

It is seen from Eqs. (64), (65), and (67) that the shapes, distributions, and orientations of the inclusions are characterized by parameters $g^{(r)}, g^{(V)}$, and $\Lambda$ for the considered heterogeneous medium. If we let $\Lambda=0$ and $g^{(V)}=2 / 3$, Eqs. (64), (65), and (67) reduce to Eqs. (47) and (48) for the random orientation; if we let $\Lambda=\infty$, they reduce to Eqs. (35) and (36) for the aligned orientation.

According to Shafiro and Kachanov, ${ }^{16}$ a large value of $\Lambda$ corresponds to the case of a slightly perturbed aligned orientation, in which case Eq. (67) becomes

$$
R_{1} \simeq \frac{3}{\Lambda^{2}}, \quad R_{2} \simeq 1-\frac{6}{\Lambda^{2}} .
$$

A small value of $\Lambda$ corresponds to the case of a weakly expressed orientational preference, and Eq. (67) becomes

$$
R_{1} \simeq \frac{1}{3}-\frac{\Lambda}{18}, \quad R_{2}=\frac{1}{3}+\frac{\Lambda}{9} .
$$

It is noted that Eq. (68) for the slightly perturbed aligned orientation contains $1 / \Lambda^{2}$ and no terms linear in $1 / \Lambda$. This indicates low sensitivity of the effective conductivities to deviations from the strictly aligned orientations. In contrast, Eq. (69) for weakly expressed orientational preference is lin- 
ear in $\Lambda$, indicating a high sensitivity of the conductivities to nonrandomness. ${ }^{16}$

The effective conductivities of heterogeneous media containing spheroidal inclusions with orientational distributions considered in this section can also be expanded in terms of the volume fraction $f^{(r)}$. If the terms higher than the second order in the volume fraction of the inclusions are ignored, Eqs. (64) and (65) can be expressed as

$\bar{\mu}_{T}=\mu^{(1)}+\mu^{(1)}\left(\left\{k_{1}^{(r)}\right\}+R_{1}\left\{k_{2}^{(r)}\right\}\right)+\frac{\mu^{(1)} g^{(V)}}{2}\left(\left\{k_{1}^{(r)}\right\}+R_{1}\left\{k_{2}^{(r)}\right\}\right)^{2}$,

$$
\begin{aligned}
\bar{\mu}_{L}= & \mu^{(1)}+\mu^{(1)}\left(\left\{k_{1}^{(r)}\right\}+R_{2}\left\{k_{2}^{(r)}\right\}\right) \\
& +\mu^{(1)}\left(1-g^{(V)}\right)\left(\left\{k_{1}^{(r)}\right\}+R_{2}\left\{k_{2}^{(r)}\right\}\right)^{2} .
\end{aligned}
$$

If the terms higher than the first order in the volume fraction of the inclusions are ignored, Eqs. (70) and (71) reduce to Eq. (46) in the paper of Shafiro and Kachanov. ${ }^{16}$ Like the formulas for aligned and randomly oriented inclusions, Eqs. (70) and (71) with the respective third terms neglected on their right-hand sides can be regarded as the generalized Maxwell formulas for multiple spheroidal inclusions with orientational distributions. Therefore, Eqs. (43), (55), (70), and (71) with the respective third terms neglected on their right-hand sides can be regarded as the generalized Maxwell formulas for aligned, randomly oriented, and orientational multiple inclusions. These results have the same degree of accuracy as those of Eqs. (1), (2), (10), and (25) in the paper of Giordano ${ }^{14}$ for spheres, circular cylinders, aligned ellipsoids, and randomly oriented ellipsoids. Thus Maxwell's theory is extended to cover a much wider range.

\section{Cavities and cracks}

Heterogeneous media containing cavities and cracks exhibit peculiar properties and thus deserve special attention. Here, we consider first a heterogeneous medium containing aligned spheroidal cavities and parallel circular cracks; the cavities and cracks have the same spheroidal distribution. Such a heterogeneous medium will be transversely isotropic. The transversely isotropic conductivities can be obtained from Eqs. (37), (38), and (42),

$$
\begin{gathered}
\bar{\mu}_{T}=\mu^{(1)}-\mu^{(1)}\left\{\left[\sum_{r=2}^{M} f^{(r)}\left(1-\frac{g^{(r)}}{2}\right)^{-1}\right]^{-1}+\frac{g^{(V)}}{2}\right\}^{-1}, \\
\bar{\mu}_{L}=\mu^{(1)}-\mu^{(1)}\left[\left(\sum_{r=2}^{M} \frac{f^{(r)}}{g^{(r)}}+\frac{8}{3} \rho\right)^{-1}+1-g^{(V)}\right]^{-1},
\end{gathered}
$$

where $M$ is the phase number of the spheroidal cavities and $\rho=(1 / V) \sum_{r=2}^{P}\left(a^{(r)}\right)^{3}$ is the scalar crack density of the circular cracks of $P$ kinds.

For a heterogeneous medium with spherical cavities and parallel circular cracks of the same size, Eqs. (72) and (73) reduce to

$$
\begin{gathered}
\bar{\mu}_{T}=\mu^{(1)}-\mu^{(1)}\left(\frac{2}{3 f_{s}}+\frac{g^{(V)}}{2}\right)^{-1}, \\
\bar{\mu}_{L}=\mu^{(1)}-\mu^{(1)}\left[\left(\frac{3}{2} f_{s}+\frac{8}{3} \rho\right)^{-1}+1-g^{(V)}\right]^{-1},
\end{gathered}
$$

where $f_{s}$ is the volume fraction of the spherical cavities.

Next we consider a heterogeneous medium containing randomly oriented and distributed spheroidal cavities and circular cracks. The isotropic conductivity can be obtained from Eqs. (47), (49), and (54),

$$
\bar{\mu}=\mu^{(1)}-\mu^{(1)}\left\{\left[\frac{1}{3} \sum_{r=2}^{M} f^{(r)}\left(\frac{4}{2-g^{(r)}}+\frac{1}{g^{(r)}}\right)+\frac{8}{9} \rho\right]^{-1}+\frac{1}{3}\right\}^{-1} .
$$

For a heterogeneous medium containing spherical cavities and circular cracks, Eq. (76) reduces to

$$
\bar{\mu}=\mu^{(1)}-\mu^{(1)}\left[\left(\frac{3}{2} f_{s}+\frac{8}{9} \rho\right)^{-1}+\frac{1}{3}\right]^{-1} .
$$

In the following, we shall illustrate the predictions of the present scheme for various kinds of heterogeneous medium and compare the predictions with those of other schemes and with experiments.

\section{NUMERICAL RESULTS}

\section{A. Conductivities of two-phase heterogeneous media}

Weber et al. ${ }^{30,31}$ measured the effective electrical conductivity of a heterogeneous medium containing randomly oriented nonconducting angular or equiaxed inclusions. In their paper, they approximated the angular inclusions by oblate spheroids with aspect ratio 3.634 and gave the theoretical predictions using the generalized self-consistent method, ${ }^{32}$ the Mori-Tanaka method (MTM), ${ }^{29}$ and the differential method (DM) ${ }^{31}$ The comparison between the theoretical and experimental results shows that the MTM overestimates significantly the effective electrical conductivity, the GSCM overestimates it slightly, and the differential method agrees well with the experimental data. ${ }^{31}$ Both the GSCM and the differential method are implicit. Here, we compare the theoretical predictions obtained from the present scheme with the above-mentioned theoretical and experimental ones, and the comparison is shown in Fig. 2. In the present scheme, the distribution of the inclusions is taken as spherical. It can be seen from Fig. 2 that the present simple and explicit prediction agrees well with the experimental data. However, for the heterogeneous medium containing nonconducting equiaxed inclusions whose shape can be treated as close to spherical, the effective conductivity obtained by the present scheme, which is equal to that predicted by MTM, ${ }^{29} \mathrm{GSCM},{ }^{32}$ and Hashin-Shtrikman upper bound, ${ }^{17}$ is slightly higher than the experimental data (cf. Fig. 3 in the paper of Weber et al. ${ }^{31}$ ).

Next, we illustrate the present scheme on heterogeneous media containing randomly oriented spheroidal inclusions or aligned spheroidal inclusions. For the former isotropic medium, the effective conductivities obtained by the Hashin- 


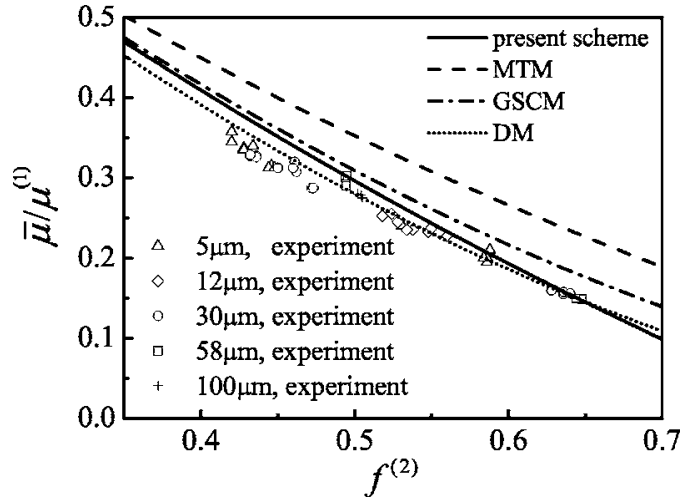

FIG. 2. Comparison of the experimental data of the effective electrical conductivity of a heterogeneous medium containing randomly oriented angular nonconducting inclusions with theoretical predictions given by the GSCM, MTM, the differential method (DM), and the present scheme. The discrete points in the figure denote the experimental data for inclusions of different sizes; $f^{(2)}$ is the volume fraction of the randomly oriented nonconducting angular inclusions.

Shtrikman bounds, ${ }^{17}$ by Giordano, ${ }^{14}$ and by the present scheme are shown in Fig. 3. The parameters used for the numerical calculations are $\gamma^{(2)}=0.2,1,2$ and $\mu^{(2)}=10 \mu^{(1)}$. The results obtained from the present scheme are close to those of Giordano ${ }^{14}$ in the range $f^{(2)}=0-0.5$, and they are both within the Hashin-Shtrikman bounds. It is noted that the present scheme coincides with the Hashin-Shtrikman lower bound for $\gamma^{(2)}=1$. For the latter heterogeneous medium with aligned spheroidal inclusions, in the numerical calculations, we assume that the inclusions have a spheroidal distribution aligned with the inclusions, and the aspect ratio of the distribution spheroid is the same as that of the inclusions. We consider the aspect ratios $\gamma^{(2)}=10,0.1$, with $\mu^{(2)}=10 \mu^{(1)}$. The transverse and longitudinal conductivities obtained by Giordano ${ }^{14}$ and the present scheme are shown in Figs. 4 and 5 . It can be seen that the results predicted by the present scheme and those by Giordano ${ }^{14}$ are fairly close in the range $f^{(2)}=0-1$.

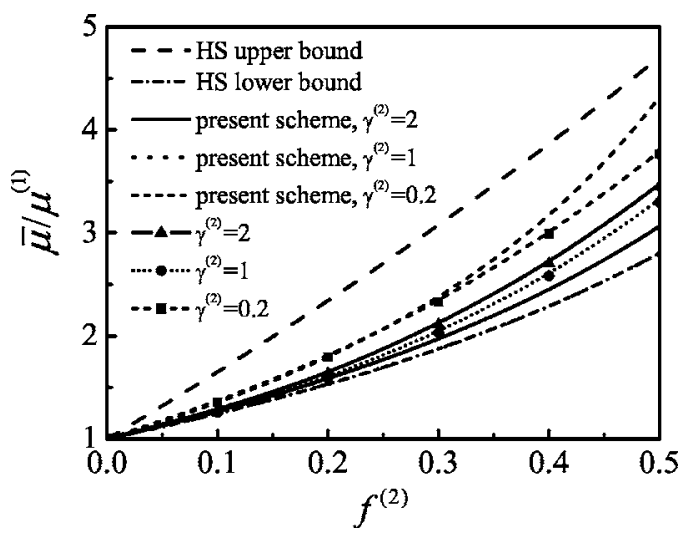

FIG. 3. Effective conductivities of heterogeneous media containing randomly oriented spheroidal inclusions with different aspect ratios and $\mu^{(2)}=10 \mu^{(1)}$ as functions of the volume fraction $f^{(2)}$ of the inclusions. Lines without symbols denote the results obtained by the present scheme, lines with symbols denote those of Giordano. ${ }^{14}$

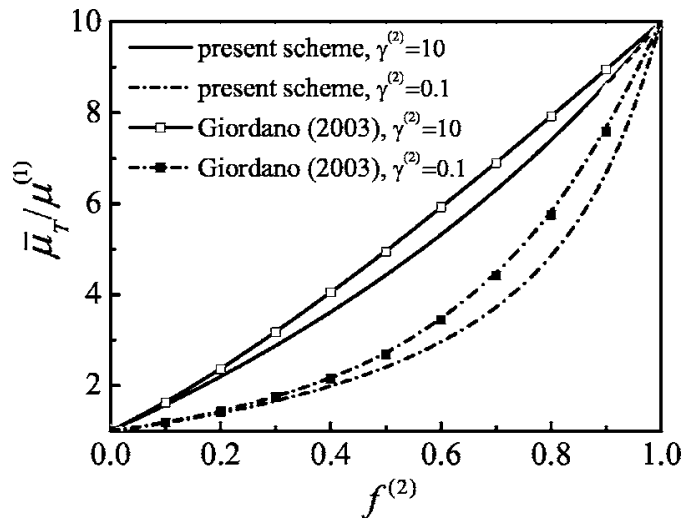

FIG. 4. Transverse conductivities of heterogeneous media containing aligned spheroidal inclusions with different aspect ratios and $\mu^{(2)}=10 \mu^{(1)}$ as functions of the volume fraction $f^{(2)}$ of the inclusions.

From Figs. 2-5, it can be seen that the effective conductivities obtained by the scheme proposed here agree well with the experimental results and those predicted by Giordano $^{14}$ for two-phase heterogeneous media containing randomly oriented spheroidal inclusions (Figs. 2 and 3), and two-phase heterogeneous media containing aligned spheroidal inclusions (Figs. 4 and 5). Thus, in contrast to the method of Shafiro and Kachanov, ${ }^{16}$ the present scheme can be applied to large volume fractions; in contrast to that of Giordano, ${ }^{14}$ the present scheme can be applied to heterogeneous media containing multiple inclusions. The results are always explicit and simple to use.

\section{B. Conductivities of three-phase heterogeneous media}

We illustrate next the present scheme on a heterogeneous medium with aligned ellipsoidal inclusions $\left(f^{(2)}=0.4, \mu^{(2)}\right.$ $\left.=10 \mu^{(1)}\right)$ and spherical inclusions $\left(f^{(3)}=0.05, \mu^{(3)}=5 \mu^{(1)}\right)$. The shape and orientation of the distribution ellipsoid are taken the same as those of the ellipsoidal inclusions. Thus the material has three principal conductivities $\bar{\mu}_{x} / \mu^{(1)}$, $\bar{\mu}_{y} / \mu^{(1)}$, and $\bar{\mu}_{z} / \mu^{(1)}$. The variations of $\bar{\mu}_{x} / \mu^{(1)}, \bar{\mu}_{y} / \mu^{(1)}$, and

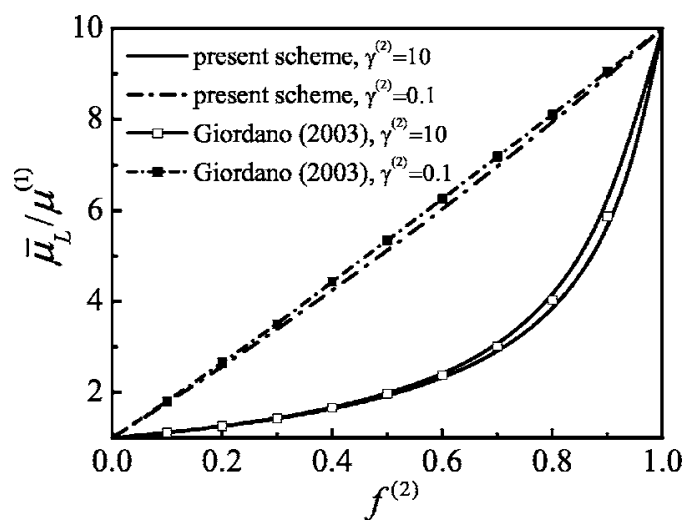

FIG. 5. Longitudinal conductivities of heterogeneous media containing aligned spheroidal inclusions with different aspect ratios and $\mu^{(2)}=10 \mu^{(1)}$ as functions of the volume fraction $f^{(2)}$ of the inclusions. 


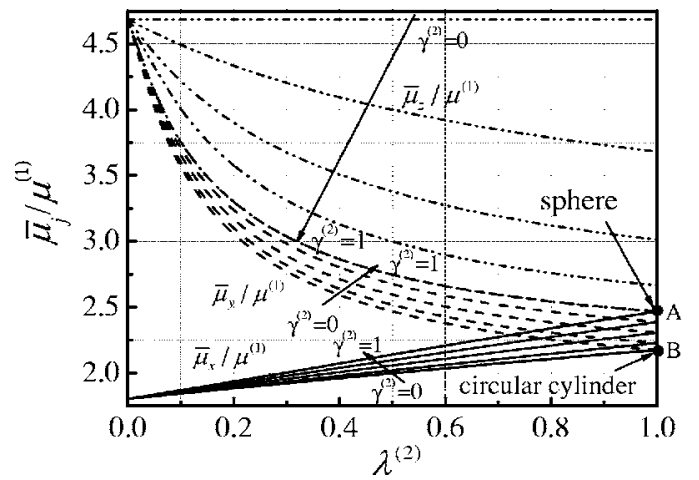

FIG. 6. Effective principal conductivities $\bar{\mu}_{j} / \mu^{(1)}(j=x, y, z)$ of a heterogeneous medium containing aligned ellipsoidal inclusions $\left(f^{(2)}=0.4, \mu^{(2)}=10 \mu^{(1)}\right)$ and spherical inclusions $\left(f^{(3)}=0.05, \mu^{(3)}\right.$ $\left.=5 \mu^{(1)}\right)(x$ axis, solid line; $y$ axis, dashed line; $z$ axis, dashed line with dots). The three families of curves $\bar{\mu}_{j} / \mu^{(1)}(j=x, y, z)$ have been plotted versus parameters $\lambda^{(2)}$ and $\gamma^{(2)}\left(\gamma^{(2)}\right.$ $=0,0.25,0.5,0.75,1$ in each family of curves).

$\bar{\mu}_{z} / \mu^{(1)}$ with aspect ratios $\lambda^{(2)}$ and $\gamma^{(2)}$ are shown in Fig. 6. In the figure, point $A$, where $\lambda^{(2)}=1$ and $\gamma^{(2)}=1$, corresponds to a mixture of two kinds of sphere with different conductivities, so that the three principal conductivities are equal and given by Eq. (51); point $B$, where $\lambda^{(2)}=1$ and $\gamma^{(2)}=0$, corresponds to a mixture of spheres and aligned circular cylinders, and the two conductivities along the $x$ and $y$ axes can be obtained from Eq. (35), and that along the $z$ axis from Eq. (36).

\section{Conductivities of media with cavities and cracks}

Finally, we will illustrate the present scheme on heterogeneous media with cracks and cavities and will compare the results with those of Shafiro and Kachanov. ${ }^{16}$ The first heterogeneous medium we study contains randomly oriented and distributed oblate cavities of the same size; the effective conductivity is given by Eqs. (47) and (49). In order to compare with the results of Shafiro and Kachanov, ${ }^{16}$ we use the crack density $\rho$ to express the volume fraction $f^{(r)}\left[f^{(r)}\right.$ $\left.=4 \pi \rho /\left(3 \gamma^{(r)}\right)\right]$. The numerical results for two aspect ratios $\gamma^{(2)}=10$ and $\gamma^{(2)}=\infty$ are shown in Fig. 7 as functions of the crack density $\rho$. The results predicted by the present scheme are lower than those of Shafiro and Kachanov ${ }^{16}$ for the same crack density. The reason is that the interactions among the cavities are taken into account by the present scheme. The second medium we study contains aligned circular cracks and spherical cavities. In this case, the considered medium is transversely isotropic, and the effective conductivity is given by Eqs. (74) and (75) and depends on the shape of the distribution spheroid. Thus, we examine six different distribution spheroids with aspect ratios ranging from $\gamma^{(V)}=0.001$ to $\gamma^{(V)}=1000$. The numerical results of $\bar{\mu}_{T} / \bar{\mu}_{L}$ are shown in Fig. 8. It can be seen that the prediction of Shafiro and Kachanov $^{16}$ is close to that given by the present scheme at $\gamma^{(V)}=1 / 3$ and $\gamma^{(V)}=1 / 2$. When the distribution ellipsoid tends to the oblate shape, the results predicted by the present scheme deviate greatly from those of Shafiro and Kachanov. ${ }^{16}$

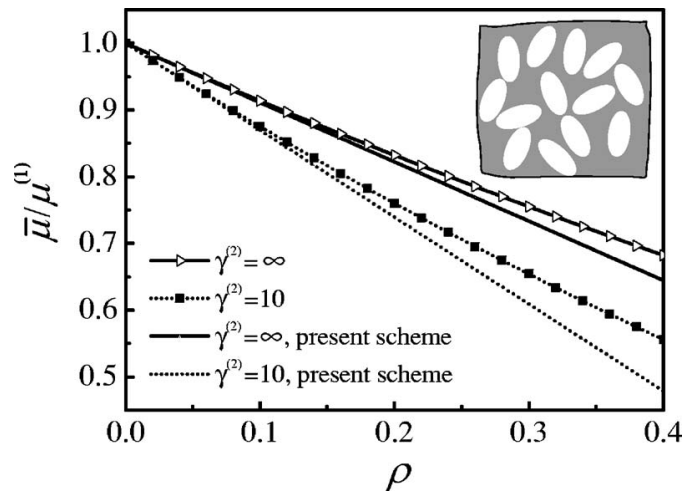

FIG. 7. Effective conductivity of a heterogeneous medium with randomly oriented oblate cavities as a function of the crack density $\rho$. Lines without symbols denote the results obtained by the present scheme, and those with symbols denote the results of Shafiro and Kachanov. ${ }^{16}$

\section{CONCLUSIONS}

A scheme is presented to predict the effective conductivities of heterogeneous media containing inclusions of various shapes, spatial distributions, and orientations. This scheme leads to completely explicit formulas for the effective conductivities in terms of three microstructural parameters characterizing the shape, distribution, and orientation of the inclusions, respectively. By expanding the conductivity tensor in terms of the volume fraction of the inclusions, it is shown that the effect of the shape of the distribution ellipsoid on the effective conductivity tensor is of higher order than the effect of the shape of the inclusions. If the terms higher than the first order in the volume fraction of the inclusion are ignored, the present scheme reduces to the noninteraction scheme of Shafiro and Kachanov. ${ }^{16}$ The present scheme generalizes the Maxwell formula to heterogeneous media containing multiple inclusions with consideration of the orientation of the inclusions. Thus, some existing formulas in the literature are shown to be the special cases of the formulas given by the present scheme. The predicted effective conductivities of heterogeneous media containing aligned ellipsoidal inclu-

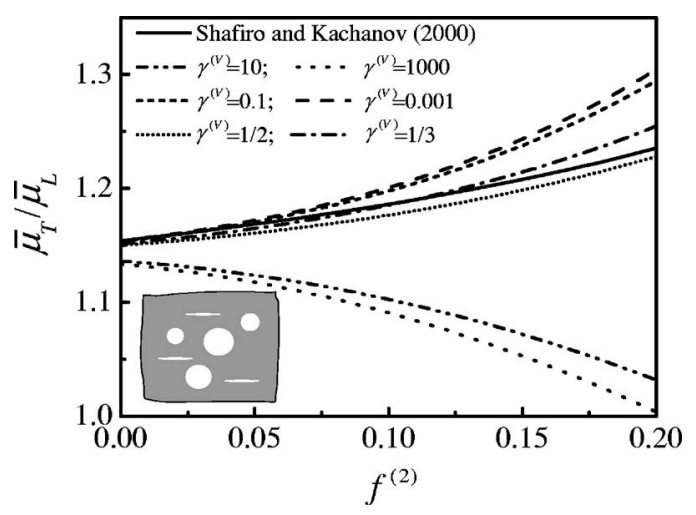

FIG. 8. Effective conductivity of a heterogeneous medium containing spherical cavities and parallel circular cracks $(\rho=0.05)$ as a function of volume fraction $f^{(2)}$ of the spherical cavities; $\gamma^{(V)}$ denotes the aspect ratio of the distribution spheroid. 
sions, randomly oriented ellipsoidal inclusions, spheroidal inclusions with orientational distributions, and mixtures of cavities and cracks are found to agree well with experimental results and predictions of other schemes. It is emphasized that in this paper, we only consider ergodic heterogeneous media, i.e., statistically homogeneous media. The present scheme cannot predict the effective conductivity close to the percolation threshold.

\section{ACKNOWLEDGMENTS}

This work was completed during Duan's visit to Cardiff University which was funded by The Royal Society. Duan, $\mathrm{Yi}$, and Wang also acknowledge the support of the National Natural Science Foundation of China under Grant No. 10525209.

\section{APPENDIX: TENSORS $\mathbf{S}^{(r)}$ AND $\mathbf{S}^{(V)}$}

For the sake of completeness, we will give the nonvanishing components of $\mathbf{S}^{(r)}$ and $\mathbf{S}^{(V)}$ for general ellipsoids. The results are expressed in terms of elliptic integrals and have been derived under the assumptions $0<a_{1}<a_{2}<a_{3}, 0<\lambda$ $=a_{1} / a_{2}<1$, and $0<\gamma=a_{2} / a_{3}<1$. For brevity, we drop the superscripts $(r)$ and $(V)$. The final expressions for $S_{11}, S_{22}$, and $S_{33}$ are, respectively,

$$
\begin{gathered}
S_{11}=\frac{1}{1-\lambda^{2}}-\frac{\lambda}{\left(1-\lambda^{2}\right) \sqrt{1-\lambda^{2} \gamma^{2}}} E(\nu, \kappa), \\
S_{22}=1-S_{11}-S_{33}, \\
S_{33}=\frac{\lambda \gamma^{2}}{\left(1-\gamma^{2}\right) \sqrt{1-\lambda^{2} \gamma^{2}}}[F(\nu, \kappa)-E(\nu, \kappa)],
\end{gathered}
$$

in which

$$
\begin{aligned}
E(\nu, \kappa)= & \int_{0}^{\nu} \sqrt{1-\kappa^{2} \sin ^{2} \omega} d \omega, \\
& F(\nu, \kappa)=\int_{0}^{\nu}\left(1-\kappa^{2} \sin ^{2} \omega\right)^{-1 / 2} d \omega,
\end{aligned}
$$

where $\nu=\arcsin \sqrt{1-\lambda^{2} \gamma^{2}}$ and $\kappa=\sqrt{1-\gamma^{2}} / \sqrt{1-\lambda^{2} \gamma^{2}}$.

For a spheroid, $a_{1}=a_{2}, \gamma=a_{1} / a_{3}=a_{2} / a_{3}$, and the final expressions for $S_{11}, S_{22}$, and $S_{33}$ are

$$
S_{33}=1-S_{11}-S_{22} \text {, }
$$

$$
\begin{aligned}
S_{11} & =S_{22}=\frac{\gamma^{2}}{2} \int_{0}^{+\infty} \frac{d \xi}{\left(\xi+\gamma^{2}\right)^{2}(\xi+1)^{1 / 2}} \\
& = \begin{cases}\frac{1}{2\left(\gamma^{2}-1\right)^{3 / 2}}\left(\gamma^{2} \arctan \sqrt{\gamma^{2}-1}-\sqrt{\gamma^{2}-1}\right), & \gamma>1, \\
\frac{1}{4\left(1-\gamma^{2}\right)^{3 / 2}}\left(2 \sqrt{1-\gamma^{2}}-\gamma^{2} \ln \frac{1+\sqrt{1-\gamma^{2}}}{1-\sqrt{1-\gamma^{2}}}\right), & \gamma<1 .\end{cases}
\end{aligned}
$$

It is noted that $\gamma>1$ corresponds to an oblate spheroid and $\gamma<1$ to a prolate spheroid.

For a penny-shaped inclusion, $\gamma \gg 1$, and Eq. (A3) reduces to

$$
S_{11}=S_{22}=\frac{\pi}{4 \gamma}, \quad S_{33}=1-\frac{\pi}{2 \gamma} .
$$

For an elliptical cylinder, $a_{3} \rightarrow \infty, \lambda=a_{1} / a_{2}$, and Eq. (A3) reduces to

$$
S_{11}=\frac{1}{\lambda+1}, \quad S_{22}=\frac{\lambda}{\lambda+1}, \quad S_{33}=0 .
$$

If $\lambda=1$, an elliptic cylinder reduces to a cylinder with a circular cross section, and the first two equations in Eq. (A5) reduce to $S_{11}=S_{22}=\frac{1}{2}$. For a sphere, $S_{11}=S_{22}=S_{33}=\frac{1}{3}$.
*Author to whom correspondence should be addressed. Electronic address: karihaloob@cardiff.ac.uk

${ }^{1}$ J. C. Maxwell, Treatise on Electricity and Magnetism (Clarendon, Oxford, 1873).

${ }^{2}$ J. W. Rayleigh, Philos. Mag. 34, 481 (1892).

${ }^{3}$ A. Einstein, Ann. Phys. 19, 289 (1905).

${ }^{4}$ L. D. Landau and E. M. Lifshitz, Electrodynamics of Continuous Media (Pergamon, Oxford, 1960).

${ }^{5}$ D. J. Bergman and D. Stroud, Solid State Phys. 46, 147 (1992).

${ }^{6}$ R. W. Zimmerman, Proc. R. Soc. London, Ser. A 452, 1713 (1996).

${ }^{7}$ K. Z. Markov, in Heterogeneous Media: Micromechanics Modeling Methods and Simulations, edited by K. Z. Markov and L. Preziosi (Birkhäuser, Boston, 2000).

${ }^{8}$ P. Ponte Castañeda, Phys. Rev. B 64, 214205 (2001).

${ }^{9} \mathrm{G}$. W. Milton, The Theory of Composites (Cambridge University Press, Cambridge, 2002).

${ }^{10}$ S. Torquato, Random Heterogeneous Materials: Microstructure and Macroscopic Properties (Springer-Verlag, New York, 2002).

${ }^{11}$ H. Fricke, Phys. Rev. 24, 575 (1924).

${ }^{12}$ L. K. H. Van Beek, Prog. Dielectr. 7, 69 (1967).

${ }^{13}$ W. Lamb, D. M. Wood, and N. W. Ashcroft, in Electrical Transport and Optical Properties of Inhomogeneous Media, edited by J. C. Garland and D. B. Tanner (AIP, New York, 1978).

${ }^{14}$ S. Giordano, J. Electrost. 58, 59 (2003).

${ }^{15}$ D. A. G. Bruggeman, Ann. Phys. 24, 636 (1935).

${ }^{16}$ B. Shafiro and M. Kachanov, J. Appl. Phys. 87, 8561 (2000).

${ }^{17}$ Z. Hashin and S. Shtrikman, J. Appl. Phys. 33, 3125 (1962).

${ }^{18}$ G. W. Milton, Phys. Rev. Lett. 46, 542 (1981).

${ }^{19}$ L. Gao and Y. Y. Huang, Eur. Phys. J. B 33, 165 (2003).

${ }^{20}$ A. V. Goncharenko, V. Z. Lozovski, and E. F. Venger, J. Phys.: Condens. Matter 13, 8217 (2001).

${ }^{21}$ A. V. Goncharenko, Phys. Rev. E 68, 041108 (2003).

22 J. R. Willis, J. Mech. Phys. Solids 25, 185 (1977).

${ }^{23}$ G. W. Milton and R. V. Kohn, J. Mech. Phys. Solids 36, 597 (1988). 
${ }^{24}$ P. Ponte Castañeda and J. R. Willis, J. Mech. Phys. Solids 43, 1919 (1995).

${ }^{25}$ P. Ponte Castañeda and P. Suquet, Adv. Appl. Mech. 34, 171 (1998).

${ }^{26}$ J. D. Eshelby, Proc. R. Soc. London, Ser. A 241, 376 (1957).

${ }^{27}$ H. Hatta and M. Taya, Int. J. Eng. Sci. 24, 1159 (1986).

${ }^{28}$ N. Kinoshita and T. Mura, Phys. Status Solidi A 5, 759 (1971).
${ }^{29}$ M. L. Dunn and M. Taya, J. Appl. Phys. 73, 1711 (1993).

${ }^{30}$ L. Weber, C. Fischer, and A. Mortensen, Acta Mater. 51, 495 (2003).

${ }^{31}$ L. Weber, J. Dorn, and A. Mortensen, Acta Mater. 51, 3199 (2003).

${ }^{32}$ T. Miloh and Y. Benveniste, J. Appl. Phys. 63, 789 (1988). 